\title{
On the continuous category in a topos
}

\author{
Tao Lu \\ School of Mathematics Science \\ HuaiBei Normal University \\ HuaiBei, AnHui, China \\ lutao7@live.com
}

\author{
Zhenzhen Zhu \\ School of Mathematics Science \\ HuaiBei Normal University \\ HuaiBei, AnHui, China \\ 995600216@qq.com
}

\begin{abstract}
The way-below relation defined on dcpos is a important concept, it is the basis for the continuity of dcpos. And a poset is also a category, so we want to generalize the concept of way-below to category and further define continous category. In this paper, we give the way-below relation on arbitrary small categories and even a topos and introduce the concept of continuous category and obtain some correspontive characterizations by mean of the diagram proof. This also shows diagram method can be used to reconstruct the classical order theory in an arbitrary elementary topos.
\end{abstract}

Keywords- coproduct; category; continuous;relation;topos

\section{INTRODUCTION}

We know that the way-below relation defined on depos is the basis for the definition of continuous dcpos as in [1] and thus the most important concept for Domain theoty and continuous lattice theory. From categorical view, a poset is a small, thin, skeletal category, a topos is a category which has finite limits and every object of has a power object. For a fixed object $A$ of category, the power object of $A$ is an object $P A$ which represents $\operatorname{Sub}\left({ }_{-} \times A\right)$, so that $\left(_{-}, P A\right) \simeq S u b\left({ }_{-} \times A\right)$ naturally. It means that for any arrow $B^{\prime} \stackrel{f}{\rightarrow} B$, the following diagram commutes, where $\varphi$ is the natural isomorphism.

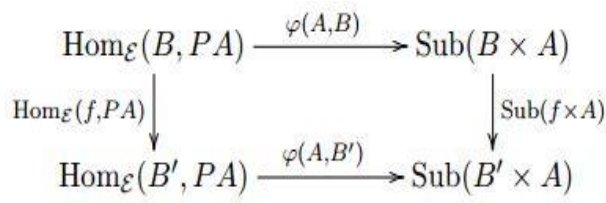

Figure 1

Mac Lane and Moerdijk, in their thorough introduction to topos theory, start their Prologue by saying -A startling aspect of topos theory is that it unifies two seemingly wholly distinct mathematical subjects: on the one hand, topology and algebraic geometry, and on the other hand, logic and set theory. Indeed, a topos can be considered both as a "generalized space" and as a "generalized universe of sets". This dual nature of topos theory is of great importance, and one can quite reasonably understand Grothendieck's name "topos" as meaning "that of which topology is the study". Mac Lane and Moerdijk are unquestionably masters of the spatial nature of toposes.
So how to define a suitable way-below ralation on arbitrary small category and further more define the concept of continuous category in a topos is interesting.

Let us review some definitons.

A locale to be a "propositional geometric theory pretending to be a space" which took the logical theory as the starting point. That is to say, the locale is the theory, but repackaged in a spatial language of points and maps instead of models and Lindenbaum algebra homomorphisms. What makes this repackaging significant is the fact that geometric logic is incomplete -in general, there are not enough standard models to account for all the

frame homomorphisms (cf. Proposition 1.4). Thus the spatial side (in terms of standard models) and the logical side (in terms of Lindenbaum algebras) become mathematically inequivalent. However, the logical side still contains good topological results; indeed, in constructive mathematics they are often better than the spatial ones. The localic repackaging makes it much easier to see this topological content. The usual definition is that a locale is a frame. We prefer to say it is the propositional geometric theory, and that it has a frame. This makes it easier to see locales as a special case of toposes, which arise from predicate geometric theories. In addition, in certain foundational schools such as predicative type theory, the frames are problematic. They are constructed using the powerset, and that is impredicative. The locale has the following properties:

And a locle is a propositional geometric theory. If the theory is $T$, we write $[T]$ for the locale. The locale $[T]$ should be conceptualized as "the space of models of $\mathrm{T}$ ".

If $X$ is a locale, then $\Omega X$ denotes its Lindenbaum algebra, a frame.

The opens of $X$ are the elements of $\Omega X$.

If $X$ and $Y$ are locales, then a map $f: X \rightarrow Y$ is a frame homomorphism. $f^{*}: \Omega Y \rightarrow \Omega X$. Locales and maps form a category Loc, dual to the category Frm of frames

As a matter of fact, the category of sheaves of sets on a topological space is a topos. In particular, the category of sets is a topos. For details of the treatment of toposes and sheaves please see [2], [3], [4], [5]. For a general background on category theory please refers to [6], [7],[8],[9],[10],[11]. 


\section{WAY-BELOW RELATION ON CONTINUOUS CATEGORIES}

Throughout this paper, all categories are small category, morphisms in small category $\mathcal{L}$ is denoted by $\rightarrow$ For $x \in o b \mathcal{L}$, denote $\downarrow x=\{y \in o b \mathcal{L}: y \rightarrow x\}$.

Definition 2.1. Let $\mathcal{L}$ be a small category, $D: \mathcal{J} \rightarrow \mathcal{L}$ be a $\mathcal{J}$ diagram. $D$ is called a filtered diagram provided for every $j, j^{\prime} \in$ obJ $\mathcal{J}$, there exists $i \in$ ob $\mathcal{J}$ and $D(i) \rightarrow D(j)$, $D(i) \rightarrow D\left(j^{\prime}\right)$

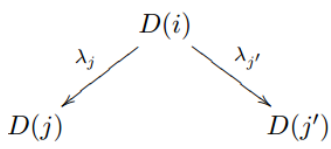

Figure 2

A directed diagram is defined dually. i.e.,for every $j, j^{\prime} \in$ ob $\mathcal{J}$, there exists $i \in$ ob $\mathcal{J}$ and $D(j) \rightarrow D(i)$, $D\left(j^{\prime}\right) \rightarrow D(i)$.

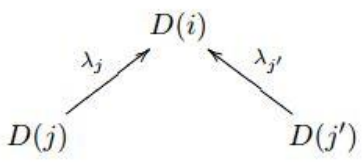

Figure 3

Definition 2.2. Let $D: \mathcal{J} \rightarrow \mathcal{L}$ be a $\mathcal{J}$ diagram. $a \in o b \mathcal{L}$

(1). If there exists a cocone over D, i.e., the following triangle commutes for any $\alpha \in \operatorname{Mor} \mathcal{L}$

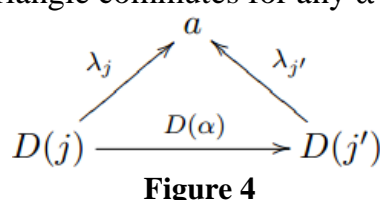

then a is called a upper bound of $D$.

(2). $a$ is called least upper bound of $D$, provided that the cocone $\left\{\lambda_{j}: D(j) \rightarrow a \mid j \in\right.$ ob $\left.\mathcal{J}\right\}$ is universal, i.e., for any cocone

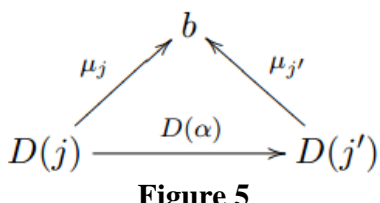

there exists an unique morphism $a \rightarrow b$ such that the following diagram commutes for any $j \in \mathrm{ob} \mathcal{J}$.

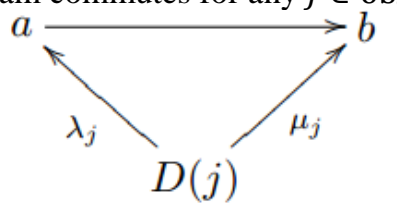

Figure 6

We denote $a$ by $\vee D$ or colim $D$. In particular, if $\mathcal{J}$ is a full subcategory of $\mathcal{L}$ then $a$ is denoted by $\vee \mathcal{J}$ or $\operatorname{colim} \mathcal{J}$. Definition 2.3. Let $\mathcal{L}$ be a small category, $\mathcal{L}$ is called directed complete provided that each directed diagram $D: \mathcal{J} \rightarrow \mathcal{L}$ has least upper bound.

Definition 2.4. Let $\mathcal{L}$ be directed complete category. $x, y \in \mathrm{ob} \mathcal{L}$, we say $x$ is way below $y$, in symbols, $x \Rightarrow y$ iff for each directed diagram $D: \mathcal{J} \rightarrow \mathcal{L}$, if there exists $y \rightarrow \operatorname{colim} D$, then we have $d_{i} \in$ ob $\mathcal{L}$ with $x \rightarrow d_{i}$.

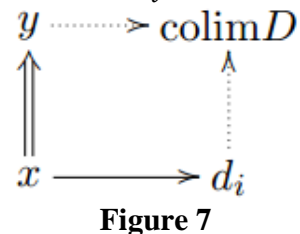

Proposition 2.1. Let $\mathcal{L}$ be a cocomplete category, i.e., $\mathcal{L}$ has all colimits, the following statements hold for $u, x, y, z \in \mathrm{ob} \mathcal{L}$

(1). $x \Rightarrow y$ implies $x \rightarrow y$;

(2). $u \rightarrow x \Rightarrow y \rightarrow z$ implies $u \rightarrow z$;

(3). $x \Rightarrow z$ and $y \Rightarrow z$ together imply $x \otimes y \Rightarrow z$, where $x \otimes y$ is the coproduct of $x$ and $y$.

(4). $0 \Rightarrow x$.

Proof.

(1). Take $D=\{b\}$, then $a \rightarrow b$ is obvious.

(2). From the following diagram, (2) can be obtained easily.

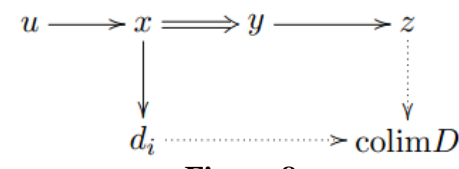

Figure 8

(3). Suppose there exists $z \rightarrow \operatorname{colim} D$ for directed diagram $D$, so there exists $d_{x} \rightarrow \operatorname{colim} D, d_{y} \rightarrow \operatorname{colim} D$, such that $x \rightarrow d_{x}, y \rightarrow d_{y}$, since $x \Rightarrow z$ and $y \Rightarrow z$. By the universal property of colimit, then $x \otimes y \rightarrow d_{x} \otimes d_{y} \rightarrow$ colim $D$. Thus $x \otimes y \Rightarrow z$.

(4). 0 is initial object, so the result is obvious.

We write $\Downarrow x=\{u \in o b \mathcal{L}: u \Rightarrow x\}, \Uparrow x=\{v \in$ $o b \mathcal{L}: x \Rightarrow v\}$.

Definition 2.5. Let $\mathcal{L}$ be a directed complete category. $\mathcal{L}$ is called a continuous category if $\{b \mid b \Rightarrow a\}$ is a directed diagram and $a=\coprod\{b \mid b \Rightarrow a\}$ for all $a \in o b \mathcal{L}$.

Lemma 2.1. In a directed complete category $\mathcal{L}$, the following conditions are equivalent:

(1). $x \Rightarrow y$

(2). $y \rightarrow \bigsqcup X$ and $X \subseteq o b \mathcal{L}$ implies the existence of a finite subset $A \subseteq X$ with $x \rightarrow \amalg A$.

Lemma 2.2. In a category $\mathcal{L}$, the following conditions are equivalent:

(1). $x \Rightarrow y$

(2). $x \in I$ for every ideal diagram $I$ of $\mathcal{L}$ such that $y \rightarrow \bigsqcup I$.

Proof.

$(1) \Rightarrow(2)$ is immediate from the definition 2.4.

(2) $\Rightarrow(1)$. Assume (2) and let $D$ be a directed diagram with $y \rightarrow \amalg D$. Then $I=\downarrow D$ is an ideal, and $y \rightarrow \bigsqcup D=\bigsqcup I$, Then $x \in I$, i.e., there is a $d \in D$ (implies $d \rightarrow \bigsqcup D$ ) such that $x \rightarrow d$. Hence $x \Rightarrow y$.

Proposition 2.2. Let $\mathcal{L}$ be a continuous category.

(1). if $x \Rightarrow z$ and $z \rightarrow \amalg D$ for a directed diagram, then exists $d \rightarrow D$, such that $x \Rightarrow d$.

(2). the way-below relation satisfies the interpolation property. i.e., $x \Rightarrow z$ implies there exists $y$ such that $x \Rightarrow y \Rightarrow z$.

Proof. 
(1). Let $D$ be a directed diagram with $z \rightarrow \amalg D$, let $I=\cup\{\Downarrow d: d \in D\}$, by continuity, $I=\amalg D$, being a union of a directed family of ideals, $I$ is an ideal diagram. Hence, if $x \Rightarrow z$ then $x \in I$, which means that $x \Rightarrow d$ for some $d \rightarrow D$.

(2). $\Rightarrow z=\amalg\{c: c \Rightarrow z\}=\amalg\{\lfloor\{d: d \Rightarrow c\} \mid c \Rightarrow z\}=$ $\amalg\{d: d \Rightarrow c \Rightarrow z\}$, thus there exists $d$ with $d \Rightarrow c \Rightarrow Z$ such that $x \rightarrow d$. Hence $x \Rightarrow c \Rightarrow z$.

Definition 2.6.Let $\mathcal{L}$ be a category which have initial object, we say that a binary relation $\prec$ on $o b \mathcal{L}$ is an auxiliary relation, if it satisfying the following conditions for all $u, x, y, z$

(1). $x \prec y$ implies $x \rightarrow y$;

(2). $u \rightarrow x<y \rightarrow z$ implies $u \prec z$;

(3). $0 \prec x$.

The set of all auxiliary relation on $o b \mathcal{L}$ will be denoted by $A u x(o b \mathcal{L})$ (considered as a category endowed with inclusion order). Clearly,every auxiliary relation is transitive and the way-below relation is an auxiliary relation. So, how we can locate the auxiliary relation $\Rightarrow$ ) within $A u x(o b \mathcal{L})$ ? For a category $\mathcal{L}$, let $\operatorname{Low}(o b \mathcal{L})$ denote the set of all lower sets in $o b \mathcal{L}$.

Proposition 2.3. Let $\mathcal{L}$ be a category and let $M$ be the set of all monotone functions $s: o b \mathcal{L} \rightarrow \operatorname{Low}(o b \mathcal{L})$ satisfying $s(x) \subseteq \downarrow x$ for all $x \in o b \mathcal{L}$ - considered as a category with the morphisms $(s \rightarrow t) \Leftrightarrow(s(x) \subseteq t(x))$ for all $x \in o b \mathcal{L}$. Then the category $A u x(o b \mathcal{L})$ is equivalence to category $M$, with functor $F: \operatorname{Aux}(o b \mathcal{L}) \rightarrow M\left(\prec \rightarrow s_{\prec}=(x \rightarrow\right.$ $\{y: y \prec x\}))$ and functor $G: M \rightarrow \operatorname{Aux}(o b \mathcal{L})(s \rightarrow$ $\left.\prec_{s}\left(x \prec_{s} y \Leftrightarrow x \in s(y)\right)\right)$.

Proof. Let $\prec$ be a auxiliary relation. Then $s_{\prec} \in M$ can be easily verified. If $s \in M, \prec_{s}$ is a auxiliary relation. So, it remained to prove the equivalence, it suffices to prove $F G=1_{M}, G F=1_{A u x(o b \mathcal{L})}$. From the following commutative diagram:

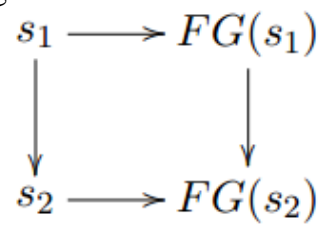

Figure 9

$F G=1_{M}$ is obtained. The rest is obvious.

From Lemma 2.2, we know that $\Downarrow x=\mathrm{n}$

$\{I \in I d(o b \mathcal{L}): x \rightarrow \bigsqcup I\}$. If we define function $m_{I:} o b \mathcal{L} \rightarrow$ $\operatorname{Low}(o b \mathcal{L})$ by

$$
m_{I}(x)=\left\{\begin{array}{l}
\downarrow x \cap I, x \rightarrow \amalg I \\
\downarrow x, x \nrightarrow \amalg I
\end{array}\right.
$$

then $m_{I} \in M$. And now we can calculate $\prod\left\{m_{I}: I \in\right.$ $I d(o b \mathcal{L})\}$ in $M$.

$$
\begin{aligned}
\prod\left\{m_{I}: I \in I d(o b \mathcal{L})\right\}(x)=\cap_{I \in I d(o b \mathcal{L})} m_{I}(x) \\
=\left(\cap_{x \rightarrow \amalg^{I}} m_{I}(x)\right) \bigcap\left(\cap_{x \rightarrow \amalg^{I}} m_{I}(x)\right) \\
=\left(\cap_{x \rightarrow \amalg^{I}} \downarrow x \cap I\right) \bigcap(\downarrow x) \\
=\cap\{I \in I d(o b \mathcal{L}): x \rightarrow \amalg I\}=\Downarrow x
\end{aligned}
$$

Definition 2.7.Let $\mathcal{L}$ be directed complete category, an auxiliary relation $\prec$ on $o b \mathcal{L}$ is called approximating iff the $\operatorname{set}\{u \in o b \mathcal{L}: u<x\}=s_{\prec}(x)$ is a directed subcategory of $\mathcal{L}$ and $x=\bigsqcup\{u \in o b \mathcal{L}: u<x\}=\bigsqcup s_{\prec}(x)$ for all $x \in o b \mathcal{L}$.

Now we can answer the question as follow:

Proposition 2.4.In a continuous category, the way-below relation $\Rightarrow$ is the smallest approximating auxiliary relation on $o b \mathcal{L}$.

Proof. From (1), and proposition 2.3, it can be obtained obviously.

We can also generalize the continuous theory to locally directed complete category and their proof is obvious.

\section{WAY-BELOW RELATION ON LOCALLY DIRECTED COMPLETE CATEGORY}

Definition 2.7. Let $\mathcal{L}$ be a small category, if for $x \in o b \mathcal{L}$, $\downarrow x=\{y \in o b \mathcal{L}: y \rightarrow x\}$ is a directed complete category, then $\mathcal{L}$ is a locally directed complete category.

Proposition 2.5. Let $\mathcal{L}$ be a locally directed complete category, then the following statements hold for $u, x, y, z \in \mathrm{ob} \mathcal{L}$

(1). $x \Rightarrow y$ implies $x \rightarrow y$;

(2). $u \rightarrow x \Rightarrow y \rightarrow z$ implies $u \rightarrow z$;

(3). If $\mathcal{L}$ has initial object 0 , then, $0 \Rightarrow z$.

Definition 2.8. Let $\mathcal{L}$ be a locally directed complete category. $\mathcal{L}$ is called a continuously locally directed complete category if $\{b \mid b \Rightarrow a\}$ is a directed diagram and $a=\amalg\{b \mid b \Rightarrow a\}$ for all $a \in o b \mathcal{L}$.

We denote $A p p(o b \mathcal{L})$ be the set of all approximative auxiliary relation on $o b \mathcal{L}$.

From Definition 2.7. we know way-below relation is a approximative auxiliary relation.

Proposition 2.6. Let $\mathcal{L}$ be a locally directed complete category, then the way-below relation is included in each approximative auxiliary relation on $o b \mathcal{L}$.

Proof. From Definition 2.6. the proof is obvious. Proposition 2.7. Let $\mathcal{L}$ be a locally directed complete category, then $\mathcal{L}$ is a continuously locally directed complete category if and only if the way-below relation is the smallest approximative auxiliary relation.

Proof. From Definition 2.8. and Proposition 2.6.the proof is obvious.

And in a locally directed complete category, the interpolation properties are also hold.

Proposition 2.8. Let $\mathcal{L}$ be a locally directed complete category and $\prec$ be a approximative auxiliary relation. For $x, z \in \mathrm{ob} \mathcal{L}$, if $x \prec z$ and $x \neq z$, then there exists $y \in \mathrm{ob} \mathcal{L}$ such that $x \rightarrow y<z$, and $x \neq y$.

Proof. From the definition of approximative auxiliary relation, the proof is obvious.

Proposition 2.9. Let $\mathcal{L}$ be a locally directed complete category, each approximative auxiliary relation has the following properties: For $x, z \in \mathrm{ob} \mathcal{L}$,

(1) Let $D: \mathcal{J} \rightarrow \mathcal{L}$ be a direct diagram and $b$ a upper bound of $D$. If $x \Rightarrow z, z \nrightarrow x$, and $z \rightarrow \amalg D$, then there exists $y \in \operatorname{ob} \mathcal{L}$, such that $x \prec y$ and $x \neq y$.

(2) If $x \Rightarrow z, z \nrightarrow x$, then there exists $y \in \mathrm{ob} \mathcal{L}$, such that $x \prec y \prec z$ and $x \neq y$.

Proof. From the definition of approximative auxiliary relation, the proof is obvious and similar.. 


\section{ACKNOWLEDGMENT}

The author would like to thank the referee for his helpful suggestions.

The work is supported by the National Natural Science Foundation (Grants No.11171156) and Science Foundation of AnHui Provience (Grant No.KJ2012Z358)

\section{REFERENCES}

[1] Gierz G , Hofmann K H. Continuous Lattice and Domains . Cambridge: Cambridge University Press, 2003.

[2] P. T Johnstone. Sketches of an Elephant: a Topos Theory Compendium . vol. 2. Oxford: Oxford University Press, 2002.

[3] L S Mac, L. Moerdijk Sheaves in Geometry and Logic: a first introduction to topos. Springer-Verlag,New York Berlin Heidelberg. 1992.

[4] A Joyal, M. Tierney. An Extension of the Galois Theory of Grothendieck. Memoirs of the American Mathematical Society, 1984.
[5] Johnstone P T, Joyal A. Continuous categories and exponentiable toposes. Journal of Pure and Applied Algebra, 1982, 25.pp. 255296.

[6] W. He Category Theory[M]. Beijing: Science Press, 2006. (in Chinese)

[7] L S. Mac, Categories for the Working Mathematician[M] Springer-verlag, New York-Berlin, 1971.

[8] Tao Lu, Hong Lu, "On the Factorization Theorem of a Monotone Morphism in a Topos" Nonlinear Maths for Uncertainty and its Appli., AISC100, Springer-Verlag Berlin Heidelberg 2011, pp. 699-706

[9] Tao Lu, Wei He, Xijuan Wang, Galois Connections in A Topos, Journal of Mathematical Research \& Exposition, Vol. 30, No. 3 May,2010,pp.381-389 . doi:10.3770/j.issn:1000-341X.2010.03.001

[10] Tao Lu, Remark on subobjects in a topos. Int. J. of Pure and Appl. Mathematics, vol 92, No. 4, 2014. 587-590.doi: 10.12732/ijpam.v92i4.13.

[11] Xijuan Wang, Wei He. On The Heyting Algebra Objects in Topos. Journal of Mathematics, vol 31, No. 6,2011,pp.15-20.

[12] He Wei, Liu Y M, Steenrod Theorem for Locales, Math Proc Camb Phil Soc, vol 124, No. 2,1998,pp.305-307.

[13] He Wei, T Plewe. Directed inverse limits of spatial locales, Proceedings of the American mathematical society vol 130, No. 10 2002, pp.2811-2814. 